\title{
Abundances in planetary nebulae: NGC 6886^
}

\author{
S. R. Pottasch ${ }^{1}$ and R. Surendiranath ${ }^{2}$ \\ 1 Kapteyn Astronomical Institute, PO Box 800, NL 9700 AV Groningen, The Netherlands \\ e-mail: pottasch@astro.rug.nl \\ 2 Indian Institute of Astrophysics, Koramangala II Block, Bangalore 560034, India
}

Received 14 September 2004 / Accepted 20 October 2004

\begin{abstract}
ISO and IUE spectra of the round-shaped planetary nebula NGC 6886 are combined with spectra in the visual wavelength region taken from the literature to obtain a complete, extinction corrected spectrum from ultraviolet to infrared wavelengths. The characteristics of the nebula and its central star are determined by various methods including photoionization modeling using CLOUDY. The results of the modeling are checked against the observational data and compared to those derived from a more classical abundance determination approach. The abundances determined are found to differ substantially from earlier results although the observations used are essentially the same, except for the inclusion of the ISO results. The reasons for this difference are discussed. Finally, the main results are interpreted in terms of the evolutionary stage of NGC 6886 and its central star.
\end{abstract}

Key words. ISM: abundances - ISM: planetary nebulae: individual: NGC 6886 - infrared: ISM

\section{Introduction}

NGC 6886 is a relatively small, round, faint planetary nebula, with an angular radius of $\sim 2.3^{\prime \prime}$. It has two "poles" where there is evidence for very low level emission extending for a further $2^{\prime \prime}$ on either side of the nebula. This is clearly shown in Fig. 1, which was taken with the Wide Field Planetary Camera 2 (WFPC2) onboard HST (see Fig. 1). The figure gives the impression of some patchiness in emission. The central star, although very faint, has been detected near the centre of the nebulosity. Heap et al. (1990) measured its continuum, deriving a visual magnitude of 18.76 , in conditions of very good seeing on Mauna Kea. They derive an extremely hot Zanstra (HeII) temperature which we will discuss later.

NGC 6886 (PK 060.1-07.7) is somewhat below the galactic plane, suggesting that this planetary nebula may be located either reasonably close or indicating a low mass progenitor star. Later in the paper a distance of about $2 \mathrm{kpc}$ is suggested. If the progenitor star is a low mass star, this eventually should be reflected in the chemical abundances derived. Statistical distances computed using different methods range between $1.5 \mathrm{kpc}$ and $5.5 \mathrm{kpc}$, which makes an estimation of its actual luminosity and mass uncertain.

Neutral hydrogen has been detected with the nebular velocity at the position of the nebula by Taylor et al. (1990) who measured the $21 \mathrm{~cm}$ line. The neutral hydrogen is undoubtedly

^ Based on observations with ISO, an ESA project with instruments funded by ESA Member States (especially the PI countries: France, Germany, The Netherlands and the UK) and with the participation of ISAS and NASA. This work has also used IUE and HST archival data.

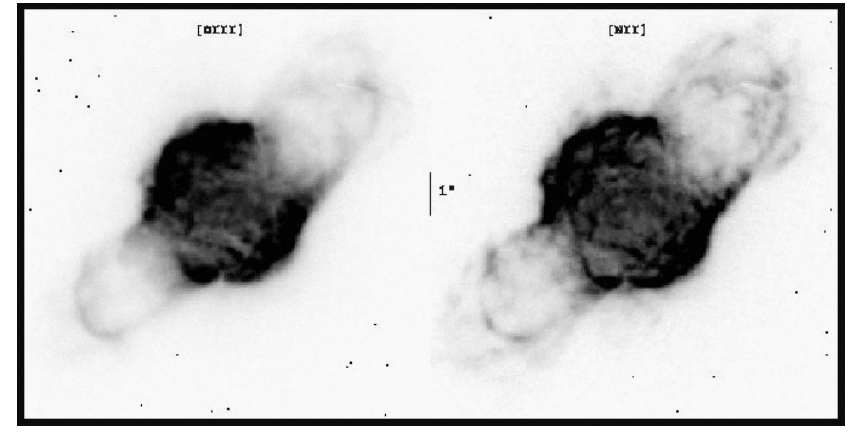

Fig. 1. HST image of the planetary nebula NGC6886 as observed through the [O III] and [N $\mathrm{NI}$ ] filters, where the overall morphology is clearly visible. North is up and East is left.

associated with the nebula and it is likely that the nebula is ionization limited.

The purpose of this paper is primarily to determine the chemical abundances for this nebula and to derive parameters like $T_{\text {eff }}, \log g$, etc., of its central star more accurately than before. This goal is achieved first by including for the first time in the analysis the ISO spectroscopic data. Second, by applying state-of-the-art photoionization modeling, to reproduce the overall spectral energy distribution and the observed nebular emission line intensities from the ultraviolet to the infrared range. The abundances in this nebula have been determined earlier by Hyung et al. (1995) but our results are very different. This is in spite of our use of almost the same observations (with the exception of the ISO data). Kwitter et al. (2001), 
using only the visible spectrum, have also determined abundances of some of the elements.

The advantages of incorporating the ISO spectroscopy in our analysis have previously been discussed (e.g. see Pottasch \& Beintema 1999; Pottasch et al. 2000, 2001; Bernard Salas et al. 2001), and can be summarized as follows.

The infrared lines originate from very low energy levels and thus give an abundance which is insensitive to the temperature in the nebula, and to possible temperature fluctuations. Furthermore, when a line originating from a high-lying energy level in the same ion is observed, it is possible to determine an effective (electron) temperature $T_{\mathrm{e}}$ at which the lines of that particular ion are formed. When $T_{\mathrm{e}}$ for many ions can be determined, it is possible to make a plot of $T_{\mathrm{e}}$ against ionization potential, which can be used to determine the $T_{\mathrm{e}}$ for ions for which only lines originating from a high energy level are observed. Use of an effective electron temperature takes into account the fact that ions are formed in different regions of the nebula. In this way possible temperature fluctuations within the nebula can be taken into account.

Use of the ISO spectra has further advantages. One of them is that the number of observed ions used in the abundance analysis is approximately doubled, which removes the need for using large "ionization correction factors", thus substantially lowering the uncertainty in the abundances derived. A further advantage is that the extinction in the infrared is almost negligible, eliminating the need to include large extinction correction factors.

A second method of improving the abundances is by using a nebular model to determine them. This has several advantages. First it provides a physical basis for the electron temperature determination. Secondly it permits abundance determinations for elements which are observed in only one, or a limited number of ionic stages. This is true of $\mathrm{Mg}, \mathrm{Ca}, \mathrm{Cl}, \mathrm{K}$ and $\mathrm{Fe}$ which could not be accurately determined without a model. A further advantage of modeling is that it provides physical information on the central star and other properties of the nebula. It thus allows one to take a comprehensive view of the nebula-star complex.

A disadvantage of modeling is that there may be more unknowns than observations and some assumptions must be made, especially concerning the geometry. In our case we will assume that the nebula is spherical and that no clumping exists. The observed round-shape form seen in Fig. 1 makes these assumptions reasonable as a first approach, although some clumping is seen. Other assumptions will be discussed in Sect. 5.

This paper is structured as follows. First the spectroscopic data are presented in Sect. 2. Section 3 discusses the radius and luminosity of the central star. Section 4 discusses a simple approach to determine the chemical composition and presents the resultant abundances. In Sect. 5 the model is presented and the assumptions made are discussed; this is followed by the abundance derivation. Section 6 compares the model spectrum with the observations. In Sect. 7 a comparison with earlier abundance determinations is made. The evolutionary state of the star-nebula system and the conclusions are given in Sects. 8 and 9.

\section{The spectrum of NGC 6886}

In the following we present the available infrared, visual and ultraviolet spectroscopic data used in our analysis. A compilation of the extinction corrected spectral line fluxes and identifications are given in Table 6.

\subsection{The ISO observations}

The ISO SWS observations were made with both the SWS01 and the SWS02 observing template (see Leech et al. 2003) The SWS02 measurements are the most accurate since more time is spent on individual lines. These measurements (TDT53701808) were centered at RA(2000) $20^{\mathrm{h}} 12^{\mathrm{m}} 42.7^{\mathrm{s}}$ and $\operatorname{Dec}(2000)+19^{\circ} 59^{\prime} 22.3^{\prime \prime}$, which is very close to the center of the nebula. The SWS01 measurements (TDT13400810), which are less accurate, cover the entire range from $2.5 \mu \mathrm{m}$ to $45 \mu \mathrm{m}$ and are therefore useful to obtain intensities for the three lines only measured by the SWS01 template ([Ar III], [S III] and [S IV]). Data reduction was carried out using ISAP (ISO Spectral Analysis Package) version 2.1. The diaphragm used was $14^{\prime \prime} \times 20^{\prime \prime}$ below $12 \mu \mathrm{m}$ and somewhat larger above this wavelength, so that the entire nebula fit within the diaphragm and was measured by the SWS. Since the SWS01 observations are about 15 to $20 \%$ weaker than the SWS02 measurements for the same lines, we have increased the SWS01 line intensities by $20 \%$. This is also in agreement with the ground based measurement of the [S IV] line intensity of Roche \& Aitken (1986) with a 7.6" diaphragm. The long wave observations were made with an LWS02 observation (TDT53701964) at essentially the same position, covering the wavelength range from $45 \mu \mathrm{m}$ to $200 \mu \mathrm{m}$. The intensity of the lines found in the spectrum is shown in Col. 3 of Table 1 . The uncertainty of the stronger lines is less than $10 \%$, while that of the weaker lines could be as large as $30 \%$. The intensities agree reasonably well with those reported by Liu et al. (2001) shown in the last column, except for the uncertain [N III] line. Also shown in the last column are the IRAS measurements (Pottasch et al. 1986) and the [S IV] measurement of Roche \& Aitken (1986).

\subsection{The IUE ultraviolet spectrum}

There are six low resolution observations on NGC 6886 taken with IUE (International Ultraviolet Explorer), all using the large entrance aperture $\left(10^{\prime \prime} \times 23^{\prime \prime}\right)$. No high resolution observations were taken. The aperture is large enough so that the emission from the entire nebula is included. We have relied on the reduction of Hyung et al. (1995) and use the intensities measured by them with the exception of the C IV line for which they give a factor of ten too low a value (due to a typographic error). Our extinction corrections are somewhat different.

We have corrected for extinction and possible misalignment by relating the ultraviolet intensities to $\mathrm{H} \beta$ in the following way. The theoretical ratio of the He II lines 1640/4686 at an electron temperature of $12500 \mathrm{~K}$ was assumed (Hummer \& Storey 1987). The other ultraviolet lines were corrected relative to $\lambda 1640 \AA$ using an extinction coefficient $c=0.70$ and the reddening curve given by Fluks et al. (1994), which is very 
Table 1. ISO spectrum of NGC 6886.

\begin{tabular}{|c|c|c|c|}
\hline Ident. & $\lambda(\mu \mathrm{m})$ & Intensity & Comparison \\
\hline H I 5-4 & 4.052 & 0.173 & \\
\hline [Mg IV] & 4.486 & 2.80 & \\
\hline [Ar VI] & 4.529 & 1.87 & \\
\hline$[\mathrm{Mg} \mathrm{v}]$ & 5.609 & 4.50 & \\
\hline$[\mathrm{Ne} \mathrm{VI}]$ & 7.652 & 0.96 & \\
\hline [Ar III] & 8.992 & 1.40 & \\
\hline [S IV] & 10.510 & 23.4 & 25. \\
\hline [Ne II] & 12.812 & 1.89 & \\
\hline$[\mathrm{Ne} \mathrm{V}]$ & 14.320 & 24.3 & \\
\hline [Ne III] & 15.554 & 38.8 & 50. \\
\hline [S III] & 18.714 & 39.3 & 40. \\
\hline$[\mathrm{Ne} \mathrm{V}]$ & 24.315 & 21.2 & \\
\hline [OIV] & 25.888 & 62.3 & \\
\hline [Ne III] & 36.010 & 3.37 & \\
\hline [O III] & 51.817 & 17.5 & 14.6 \\
\hline [N III] & 57.328 & $6.4:$ & 2.7: \\
\hline [O III] & 88.401 & 3.30 & 2.97 \\
\hline
\end{tabular}

Column 3 gives our measured values. The last column gives the IRAS measurements (Pottasch et al. 1986) below $20 \mu \mathrm{m}$, the [S IV] measurement of Roche \& Aitken (1986) and the values of Liu et al. (2001) above $50 \mu \mathrm{m}$. All intensities are in units of $10^{-12} \mathrm{erg} \mathrm{cm}^{-2} \mathrm{~s}^{-1}$. The ":" on the $[\mathrm{N} \mathrm{III]}$ line intensity indicates that the line is weak and difficult to measure.

similar to that given by Seaton (1979). The extinction coefficient used will be discussed presently. Since only a correction with respect to $\lambda 1640 \AA$ is used, it reduces the possible error considerably. The intensities are given in Table 6 .

\subsection{The visual spectrum}

NGC 6886 has been optically observed on several occasions in the last two decades. The highest resolution spectra are those of Hyung et al. (1995), who use a slit of $1.2^{\prime \prime} \times 4.0^{\prime \prime}$, which is somewhat smaller than the nebula. They measure the spectrum from $\lambda 3650 \AA$ to $10050 \AA$. The measurements of Aller $\&$ Czyzak (1979) are made with a slit of $2^{\prime \prime} \times 2^{\prime \prime}$, have a somewhat lower resolution, but extend in the ultraviolet down to $\lambda 3130 \AA$. A third set of spectra are those of Kwitter et al. (2001) who have a much lower spectral resolution, a broader slit $\left(5^{\prime \prime} \times 285^{\prime \prime}\right)$ and do not include the ultraviolet shortward of $\lambda 3700 \AA$.

All three authors reduce the spectra in a similar way. After correcting for the instrumental properties, they then determine the extinction coefficient " $c$ " such that the theoretical Balmer decrement (as determined by Hummer \& Storey 1987) is obtained. All three obtain different values of " $c$ ". Hyung et al. find $c=0.90$, Aller \& Czyzak find $c=1.08$ and Kwitter et al. find $c=0.57$. We regard it as unlikely that these differences are real and due to measurements in different parts of the nebula. More likely they result from a somewhat incorrect wavelength calibration. This mistaken correction can still give the correct Balmer decrement if a somewhat different (and incorrect) value of " $c$ " is used. Since all three result in a correct Balmer (and
Paschen) decrement, we regard the line ratios found to be "correct". We have essentially used the results of Hyung et al. for two reasons. First these measurements cover the largest part of the nebula, and secondly they are made with the highest resolution, thus minimizing the effects of possible blends. For the $\lambda 3425 \AA$ line of [Ne V] we have used the measurements of Rowlands et al. (1993). The observed fluxes are listed in Table 6.

It would have been preferred if the visual observations had been made with a diaphragm large enough to cover the entire nebula, just as the ISO and IUE observations do. There are three reasons to believe that the observations used are a good approximation to what would be seen in a larger diaphragm. First, the observations used agree with those of Kwitter et al. which cover a somewhat different part of the nebula because of the long slit they used. Secondly observations have been made by Tsamis et al. (2003) who scanned nine planetary nebulae so that an average spectrum is obtained in each case. This spectrum is not very different from spectra made at individual positions in each nebula by other authors, or by Tsamis et al. themselves in the case of IC 4191.

\subsection{Extinction}

There are several methods for estimating the extinction towards planetary nebulae. We have already mentioned the comparison of observed and theoretical Balmer decrement and the uncertainties which can result. Perhaps the most reliable method is a comparison of radio emission with $\mathrm{H} \beta$ flux. The $\log \mathrm{H} \beta$ flux is -11.31 (see Cahn et al. 1992). The $6 \mathrm{~cm}$ radio flux density is more uncertain. Gregory \& Condon (1991) find 88 mJy, Becker et al. (1991) give $85 \mathrm{mJy}$, Cahn et al. (1992) give $98 \mathrm{mJy}$. If we use $88 \mathrm{mJy}$, a value of $\mathrm{He}^{\circ}=0.069$ and $\mathrm{He}^{+}=0.038$, a value of $T_{\mathrm{e}}=12500 \mathrm{~K}$ (see below), we obtain $c=0.70$ or $E_{B-V}=0.47$. This is similar to an average obtained from the Balmer decrement measurements, but probably more accurate. This is the value which has been used in the remainder of this paper, together with the extinction curves of Seaton (1979) and Fluks et al. (1994). Finally, since NGC 6886 is a high excitation object, i.e. $F_{\mathrm{He} \text { II } 4686} \AA$ is about half of $F_{\mathrm{H} \beta}$, we corrected the unreddened $\mathrm{H} \beta$ flux for contamination by $\mathrm{He}^{+}$Pickering $8 \rightarrow 4$ transition. The correction is only $2.5 \%$. All unreddened line fluxes were normalized to this unreddened and uncontaminated $\mathrm{H} \beta$ flux as 100 units and they are shown in Table 6. The absolute values can be obtained using extinction corrected $F_{\mathrm{H} \beta}=2.34 \times 10^{-11} \mathrm{erg} \mathrm{cm}^{-2} \mathrm{~s}^{-1}$.

\section{Radius and luminosity of the central star}

The stellar radius and luminosity can be obtained from the visual observed stellar magnitude, the effective temperature and the distance of the nebula.

The distance of the nebulae is difficult to obtain accurately. By equating the $\langle\mathrm{rms}\rangle$ density with the forbidden line density (see Sect. 4.1) an uncertain value of $d=2.0 \mathrm{kpc}$ is found and this will be used when necessary throughout this paper. It has, however, an uncertainty which could be larger than $40 \%$. 
The stellar radius can now be obtained from the measured stellar magnitude of 18.76 (Heap et al. 1990) which when corrected for the extinction given earlier becomes 17.30. This leads to a stellar radius of $R_{\star} / R_{\odot}=0.029$, if blackbody radiation is assumed.

The stellar temperature was obtained both from the Zanstra method and the Energy Balance method. Assuming a blackbody, a hydrogen Zanstra temperature $T_{Z}(\mathrm{H})=145000 \mathrm{~K}$ is found. The same value $(145000 \mathrm{~K})$ is obtained for the ionized helium Zanstra temperature $T_{Z}(\mathrm{HeII})$. To determine the temperature from the Energy Balance Method, the ratio of the line intensities excited by collisions to $\mathrm{H} \beta$ must be known. It was found by adding all the collisionally excited lines in the various tables above to be 53.2. Applying the theory of Preite-Martinez \& Pottasch (1983) assuming a blackbody spectrum for the exciting star and their Case III (optically thick nebula), the resultant energy balance temperature is $T_{\mathrm{EB}}=156000 \mathrm{~K}$.

Using the above temperature $\left(T_{\mathrm{eff}}=152000 \mathrm{~K}\right)$, the stellar luminosity $L_{\star} / L_{\odot}$ is found to be 400 . It is also possible to obtain the stellar luminosity from the nebular $\mathrm{H} \beta$ luminosity, since there is a direct relationship between the number of ionizing photons and the number of $\mathrm{H} \beta$ photons, in the case in which the nebula absorbs all the ionizing photons emitted by the star. A mathematical formulation of this can be found in Pottasch \& Acker (1989). It yields the following luminosity: $L / L_{\odot}=413$. This is roughly the same value as found above and indicates that most of the ionizing photons must actually be absorbed in the nebula. However, it does not give any information about the distance, since both formulations have the same distance dependence.

\section{Chemical composition of NGC 6886 from the simplified analysis}

The method of analysis is the same as used in the papers cited in the introduction. First the electron density and the temperature as a function of the ionization potential are determined. Then the ionic abundances are determined, using the density and the temperature appropriate for the ion under consideration, together with Eq. (1). Then the element abundances are found for those elements for which a sufficient number of ionic abundances have been derived.

\subsection{Electron density}

The ions used to determine $N_{\mathrm{e}}$ are listed in the first column of Table 2. The ionization potential required to reach that ionization stage, and the wavelengths of the lines used, are given in Cols. 2 and 3 of the table. Note that the wavelength units are $\AA$ when 4 ciphers are given and microns when 3 ciphers are shown. The observed ratio of the lines is given in the fourth column; the corresponding $N_{\mathrm{e}}$ is given in the fifth column. The temperature used is discussed in the following section, but is unimportant since these line ratios are essentially determined by the density. The atomic parameters used are the same as in the earlier papers cited above. The only exception to this is the case of [O II] for which the values recently recommended by Wang et al. (2004) have been used.
Table 2. Electron density indicators in NGC 6886.

\begin{tabular}{lcccc}
\hline \hline Ion & $\begin{array}{c}\text { Ioniz. } \\
\text { pot. }(\mathrm{eV})\end{array}$ & $\begin{array}{c}\text { Lines } \\
\text { used }\end{array}$ & $\begin{array}{c}\text { Observed } \\
\text { ratio }\end{array}$ & $\begin{array}{c}N_{\mathrm{e}} \\
\left(\mathrm{cm}^{-3}\right)\end{array}$ \\
\hline$[\mathrm{S} \mathrm{II}]$ & 10.4 & $6731 / 6716$ & 2.0 & 10000 \\
{$[\mathrm{O}$ II $]$} & 13.6 & $3626 / 3729$ & 2.0 & 5200 \\
{$[\mathrm{Cl}$ III $]$} & 23.8 & $5538 / 5518$ & 2.1 & 16000 \\
{$[\mathrm{Ar}$ IV $]$} & 40.7 & $4711 / 4740$ & 0.77 & 6000 \\
\hline
\end{tabular}

Table 3. Electron temperature indicators in NGC 6886.

\begin{tabular}{lcccc}
\hline \hline Ion & $\begin{array}{c}\text { Ioniz. } \\
\text { pot. }(\mathrm{eV})\end{array}$ & $\begin{array}{c}\text { Lines } \\
\text { used }\end{array}$ & $\begin{array}{c}\text { Observed } \\
\text { ratio }\end{array}$ & $\begin{array}{c}T_{\mathrm{e}} \\
(\mathrm{K})\end{array}$ \\
\hline$[\mathrm{N} \mathrm{II}]$ & 14.5 & $5755 / 6584$ & 0.0209 & 10000 \\
{$[\mathrm{~S} \mathrm{III}]$} & 23.3 & $6312 / 18.7$ & 0.081 & 10000 \\
{$[\mathrm{~N} \mathrm{III}]$} & 29.6 & $1750 / 57.3$ & 1.5 & 10800 \\
{$[\mathrm{O} \mathrm{III}]$} & 35.1 & $4363 / 5007$ & 0.0115 & 13000 \\
{$[\mathrm{O} \mathrm{III}]$} & 35.1 & $1663 / 5007$ & 0.0227 & 12500 \\
{$[\mathrm{Ne} \mathrm{III}]$} & 41.0 & $3868 / 15.5$ & 0.86 & 12500 \\
{$[\mathrm{O} \mathrm{IV}]$} & 54.9 & $1400 / 25.9$ & 0.126 & 15000 \\
{$[\mathrm{Ne}$ IV $]$} & 63.4 & $2424 / 4725$ & $95 .:$ & 16000 \\
{$[\mathrm{Ne} \mathrm{v}]$} & 97.1 & $3425 / 24.3$ & $0.64 .:$ & 14000 \\
{$[\mathrm{Ne} \mathrm{v}]$} & 97.1 & $3425 / 14.3$ & $0.55 .:$ & 16600 \\
{$[\mathrm{Mg} \mathrm{v}]$} & 109.3 & $2784 / 5.61$ & 0.573 & 16000 \\
\hline
\end{tabular}

The ":" indicate uncertain observations.

Electron densities could not be determined from the [Ne III] and $[\mathrm{Ne} \mathrm{V}]$ line ratios. These ratios, which have a small density dependence at densities of interest for this nebula, are not useful in other nebulae either. It is thought that the problem is the determination of the atomic parameters for these lines.

The electron density appears to be about $10000 \mathrm{~cm}^{-3}$. There is no indication that the electron density varies with ionization potential in a systematic way, although only four values are determined. It is interesting to compare this value of the density with the $\langle$ rms $\rangle$ density found from the $\mathrm{H} \beta$ line. This depends on the distance of the nebula which is not accurately known, and on the angular size of the nebula. Because of the distance uncertainty, we shall turn the calculation around, and compute what the distance will be for an $\langle\mathrm{rms}\rangle$ density of $10000 \mathrm{~cm}^{-3}$ in a sphere of radius $2.3^{\prime \prime}$, that emits the $\mathrm{H} \beta$ flux given above. This yields an uncertain distance of $2.0 \mathrm{kpc}$. This value will be used in further computations in this paper.

\subsection{Electron temperature}

A number of ions have lines originating from energy levels far enough apart that their ratio is sensitive to the electron temperature. These are listed in Table 3, which is arranged similarly to the previous table. The electron temperature is found to increase as a function of ionization potential. There is some scatter. A value of $N_{\mathrm{e}}=10000 \mathrm{~cm}^{-3}$ has been used in the computations, but the temperature is in general not sensitive to the electron density. 


\subsection{Ionic and element abundances}

The ionic abundances have been determined using the following equation:

$\frac{N_{\text {ion }}}{N_{\mathrm{p}}}=\frac{I_{\text {ion }}}{I_{\mathrm{H}_{\beta}}} N_{\mathrm{e}} \frac{\lambda_{\mathrm{ul}}}{\lambda_{\mathrm{H}_{\beta}}} \frac{\alpha_{\mathrm{H}_{\beta}}}{A_{\mathrm{ul}}}\left(\frac{N_{\mathrm{u}}}{N_{\mathrm{ion}}}\right)^{-1}$

where $I_{\mathrm{ion}} / I_{\mathrm{H}_{\beta}}$ is the measured intensity of the ionic line compared to $\mathrm{H} \beta, N_{\mathrm{p}}$ is the density of ionized hydrogen, $\lambda_{\mathrm{ul}}$ is the wavelength of this line, $\lambda_{\mathrm{H}_{\beta}}$ is the wavelength of $\mathrm{H} \beta, \alpha_{\mathrm{H}_{\beta}}$ is the effective recombination coefficient for $\mathrm{H} \beta, A_{\mathrm{ul}}$ is the Einstein spontaneous transition rate for the line, and $N_{\mathrm{u}} / N_{\text {ion }}$ is the ratio of the population of the level from which the line originates to the total population of the ion. This ratio has been determined using a five level atom.

The results are given in Table 4, where the first column lists the ion concerned, and the second column the line used for the abundance determination. The third column gives the intensity of the line used relative to $\mathrm{H} \beta=100$. The fourth column gives the electron temperature used, which is a function of the ionization potential and is found from Table 3. The ionic abundances, are in the fifth column, while the sixth column gives the Ionization Correction Factor (ICF).This has been determined empirically. Notice that the ICF is unity for helium, carbon, nitrogen, oxygen and neon because all important stages of ionization have been observed. The ICFs for the other elements have been determined by comparing the observed ionization stages as a function of ionization potential with those elements where all important ionization stages are present, especially nitrogen and neon. The four ionization stages in argon are the most important stages and thus justify the use of an ICF close to unity. For sulfur the importance of the missing ionization stages is difficult to judge; therefore an empirical ICF is very uncertain. This is less true of magnesium and chlorine, which might still be uncertain by a factor of two. Only one stage of ionization has been observed in silicon and calcium for which only a model approach can give a trustworthy solution.

Iron was not attempted with this approach. The element abundances are given in the last column of the table.

The helium abundance has been derived using the theoretical work of Benjamin et al. (1999). For recombination of singly ionized helium, most weight is given to the $\lambda 5875 \AA$ line, because the theoretical determination of this line is the most reliable.

The abundances in NGC 6886 are in general not very similar to solar abundances. The oxygen abundance is almost solar. Neon, argon and chlorine are within a factor of two of solar. Nitrogen and carbon are substantially higher. We shall discuss the meaning of these abundances later.

\subsubsection{Recombination line abundances}

There are two possible recombination lines in the spectrum for C II. These are the lines at $\lambda 4267 \AA$, and at $\lambda 1761 \AA$. Using the observed ratio of these lines to $\mathrm{H} \beta$ of $4.3 \times 10^{-3}$ and $1.2 \times 10^{-1}$ respectively, and the effective recombination coefficient to form these lines given by Davey et al. (2000) at at an electron temperature of $T=12500$, we obtain a value of
Table 4. Ionic concentrations and chemical abundances in NGC 6886. Wavelength in Angstrom for all values of $\lambda$ above 1000, otherwise in $\mu \mathrm{m}$.

\begin{tabular}{|c|c|c|c|c|c|c|}
\hline Ion & $\lambda$ & $I$ & $T$ & $N_{\text {ion }} / N_{\mathrm{p}}$ & ICF & $N_{\mathrm{el} .} / N_{\mathrm{p}}$ \\
\hline $\mathrm{He}^{+}$ & 5875 & 9.6 & 11000 & 0.069 & & \\
\hline $\mathrm{He}^{++}$ & 4686 & 45. & 12500 & 0.038 & 1 & 0.107 \\
\hline $\mathrm{C}^{+}$ & 2325 & 129. & 10000 & $2.35(-4)$ & & \\
\hline $\mathrm{C}^{++}$ & 1909 & 628. & 10500 & $1.08(-3)$ & & \\
\hline $\mathrm{C}^{+3}$ & 1548 & 502. & 14000 & $1.14(-4)$ & 1 & $14.3(-4)$ \\
\hline $\mathrm{N}^{+}$ & 6584 & 225. & 10200 & $4.3(-5)$ & & \\
\hline $\mathrm{N}^{++}$ & 1750 & 41. & 11000 & $2.63(-4)$ & & \\
\hline $\mathrm{N}^{++}$ & 57.3 & 27.4 . & 11000 & $3.06(-4)$ & & \\
\hline $\mathrm{N}^{+3}$ & 1485 & 36.8 & 14000 & $5.68(-5)$ & & \\
\hline $\mathrm{N}^{+4}$ & 1239 & 64. & 16000 & $2.76(-5)$ & 1 & $4.2(-4)$ \\
\hline $\mathrm{O}^{+}$ & 3727 & 150. & 10300 & $1.0(-4)$ & & \\
\hline $\mathrm{O}^{+}$ & 2470 & 33.5 & 10300 & $1.7(-4)$ & & \\
\hline $\mathrm{O}^{++}$ & 5007 & 1500. & 12000 & $3.49(-4)$ & & \\
\hline $\mathrm{O}^{++}$ & 51.8 & 74.8 & 12000 & $4.90(-4)$ & & \\
\hline $\mathrm{O}^{+3}$ & 25.8 & 266. & 15500 & $1.69(-4)$ & & \\
\hline $\mathrm{O}^{+3}$ & 1400 & 33.5 & 15500 & $1.1(-4)$ & 1 & $6.5(-4)$ \\
\hline $\mathrm{Ne}^{+}$ & 12.8 & 8.08 & 11000 & $1.2(-5)$ & & \\
\hline $\mathrm{Ne}^{++}$ & 15.5 & 166. & 12500 & $1.14(-4)$ & & \\
\hline $\mathrm{Ne}^{++}$ & 3869 & 143. & 12500 & $1.36(-4)$ & & \\
\hline $\mathrm{Ne}^{+3}$ & 2424 & 116. & 16000 & $3.84(-5)$ & & \\
\hline $\mathrm{Ne}^{+4}$ & 24.3 & 90.6 & 16500 & $2.37(-5)$ & & \\
\hline $\mathrm{Ne}^{+4}$ & 14.3 & 104. & 16500 & $1.32(-5)$ & & \\
\hline $\mathrm{Ne}^{+5}$ & 7.65 & 4.1 & 16500 & $1.49(-8)$ & 1 & $2.0(-4)$ \\
\hline $\mathrm{S}^{+}$ & 6731 & 12.2 & 10000 & $1.0(-6)$ & & \\
\hline $\mathrm{S}^{++}$ & 6312 & 2.9 & 10500 & $4.27(-6)$ & & \\
\hline $\mathrm{S}^{++}$ & 18.7 & 32.1 & 10500 & $5.19(-6)$ & & \\
\hline $\mathrm{S}^{+3}$ & 10.5 & 90. & 12000 & $3.59(-6)$ & 1.15 & $1.0(-5)$ \\
\hline $\mathrm{Ar}^{++}$ & 7136 & 24. & 11500 & $1.71(-6)$ & & \\
\hline $\mathrm{Ar}^{++}$ & 8.99 & 5.98 & 11500 & $6.14(-7)$ & & \\
\hline $\mathrm{Ar}^{+3}$ & 4740 & 7.6 & 12000 & $0.99(-6)$ & & \\
\hline $\mathrm{Ar}^{+4}$ & 7005 & 1.8 & 16000 & $1.87(-7)$ & & \\
\hline $\mathrm{Ar}^{+5}$ & 4.53 & 7.95 & 16000 & $1.52(-7)$ & 1.02 & $2.1(-6)$ \\
\hline $\mathrm{Cl}^{+}$ & 8580 & 0.25 & 10300 & $2.19(-8)$ & & \\
\hline $\mathrm{Cl}^{++}$ & 5538 & 1.0 & 10700 & $1.01(-7)$ & & \\
\hline $\mathrm{Cl}^{+3}$ & 8046 & 0.86 & 12500 & $6.07(-8)$ & 1.2 & $2.2(-7)$ \\
\hline $\mathrm{Mg}^{+}$ & 2798 & 23. & 10000 & $7.9(-7)$ & & \\
\hline $\mathrm{Mg}^{+3}$ & 4.49 & 12. & 16000 & $5.73(-6)$ & & \\
\hline $\mathrm{Mg}^{+4}$ & 5.61 & 19.2 & 16000 & $5.18(-6)$ & 1.7: & 2.0(-5): \\
\hline $\mathrm{Si}^{++}$ & 1888 & 19.8 & 10100 & $8.0(-7)$ & & \\
\hline $\mathrm{Ca}^{+5}$ & 5309 & 0.18 & 13000 & $2.1(-8)$ & & \\
\hline
\end{tabular}

Intensities $(I)$ given with respect to $\mathrm{H} \beta=100$. The colon “:” indicates an uncertain correction for the missing ionization stages.

$\mathrm{C}^{++}=4.8 \times 10^{-4}$ and $2.1 \times 10^{-2}$. This compares with the value of $1.08 \times 10^{-3}$ obtained from the collisionally excited line at $\lambda 1909 \AA$. The value obtained from the $\lambda 4267 \AA$ line is a factor of two lower, but because it is so weak it may not be very accurate. The value obtained from the $\lambda 1761 \AA$ line is a factor of 20 higher. The effective recombination coefficients for these two lines are not consistent. It is possible that either 
the $\lambda 1761 \AA$ line is due to some other ion or the effective recombination coefficient is wrong.

\section{Model}

In order to obtain as nearly correct a model as possible, the star as well as the nebula must be considered. Let us first consider the star. There are several ways to obtain a reasonable estimate of the stellar temperature. In Sect. 3 we have already considered the temperature determination on the assumption that the star radiates as a blackbody. For the model we used a NLTE model computed by Rauch (2003) with heavy element opacity added. The best agreement with the observed ionization was obtained with a model which had a temperature $T_{\text {eff }}=159000 \mathrm{~K}$. This temperature is consistent with the values found in Sect. 3 .

Modeling the nebula-star complex will allow characterizing not only the central star's temperature but other stellar parameters as well (i.e., $\log g$ and luminosity). It can determine distance and other nebular properties, especially the composition, including the composition of elements which are represented by a single stage of ionization, which cannot be determined by the simplified analysis above. This method can take into account presence of dust and molecules in the nebular material and thus is very comprehensive. While the line ratio method is simple and fast, the ICFs rest on uncertain physics. To this end, modeling serves as an effective means and the whole set of parameters is determined in a unified way, assuring self consistency. Finally, in this way one gets a good physical insight into the PN, the method and the observations.

It is with this in mind that we have constructed a photoionization model for NGC 6886 with the code Cloudy, using the latest version 96.04 i.e., 96 beta 5 (Ferland 2001).

\subsection{Assumptions in the model}

We have considered only gas phase abundances of various elements seen in the spectra (Table 6). We examined the HST images of NGC 6886 (Fig. 1) and found that the image is nearly circular if we may ignore the very low intensity "caps". We have determined the angular diameter from these images as $4.3^{\prime \prime}$, and use this value for modeling. It may be noted that several earlier determinations (Acker et al. 1992; Hyung et al. $1995)$ give a somewhat larger diameter $\left(5.5^{\prime \prime}\right)$. The distribution of light is not entirely smooth and there is some clumpiness in the images. In spite of this we have used a constant density model as a first approximation. We worked with a spherically symmetric and static nebula. All the properties derived for NGC 6886 in Sects. 1-4 have been used as start-up values in the modeling. For elements whose abundances were not known a priori, typical PN abundances have been inserted. To represent the central star energy distribution in the model, we chose the Rauch (2003) model atmospheres. These NLTE model atmospheres include metal-line blanketing, i.e., they include light metals as well as the iron-group ( $\mathrm{Sc}, \mathrm{Ti}, \mathrm{V}, \mathrm{Cr}, \mathrm{Mn}, \mathrm{Fe}, \mathrm{Co}$ and $\mathrm{Ni}$ ) opacities with millions of lines, following Kurucz's line lists. They have been computed for both solar and halo abundances of the central star. Therefore they are ideal for our purpose.
Table 5. Parameters representing the best-fit model.

\begin{tabular}{|c|c|c|c|c|}
\hline Parameter & \multicolumn{4}{|l|}{ Value } \\
\hline$C S P N$ & & & & \\
\hline Model atmosphere & \multicolumn{4}{|c|}{ Rauch (2003) } \\
\hline$T_{\text {eff }}$ & \multicolumn{4}{|c|}{$159000 \mathrm{~K}$} \\
\hline $\log g$ & \multicolumn{4}{|c|}{7.0} \\
\hline Luminosity & \multicolumn{4}{|l|}{$690 L_{\odot}$} \\
\hline \multicolumn{5}{|l|}{ Nebula } \\
\hline Const. density & \multicolumn{4}{|c|}{$N_{\mathrm{H}}=7.86 \times 10^{3} \mathrm{~cm}^{-3}$} \\
\hline \multirow{8}{*}{ Abundance } & $\mathrm{H}$ & $\mathrm{He}$ & $\mathrm{C}$ & $\mathrm{N}$ \\
\hline & 12.000 & 11.033 & 9.283 & 8.665 \\
\hline & $\mathrm{O}$ & $\mathrm{Ne}$ & $\mathrm{Mg}$ & $\mathrm{Si}$ \\
\hline & 8.892 & 8.230 & 7.541 & 7.322 \\
\hline & $\mathrm{S}$ & $\mathrm{Cl}$ & Ar & $\mathrm{K}$ \\
\hline & 7.000 & 5.342 & 6.301 & 5.079 \\
\hline & $\mathrm{Ca}$ & $\mathrm{Fe}$ & & \\
\hline & 4.079 & 5.699 & & \\
\hline Size & \multicolumn{4}{|c|}{ 4".3 (diameter) } \\
\hline Distance & \multicolumn{4}{|c|}{$2.55 \mathrm{kpc}$} \\
\hline Inner radius & \multicolumn{4}{|c|}{$5.00 \mathrm{e} 15 \mathrm{~cm}(\sim 0.00162 \mathrm{pc})$} \\
\hline Outer radius & \multicolumn{4}{|c|}{$8.201 \mathrm{e} 16 \mathrm{~cm}(\sim 0.02658 \mathrm{pc})$} \\
\hline Filling factor & \multicolumn{4}{|c|}{1} \\
\hline
\end{tabular}

\subsection{Results}

The general method of application of the code Cloudy to model NGC 6886 is the same as in Surendiranath et al. (2002); we ran a number of models and each time the output was carefully scrutinized before running the next model with changed input parameters. We tried to match the observed spectral fluxes of about 70 lines. We relied on physical intuition rather than any of the optimization techniques provided in Cloudy. We succeeded in getting a reasonably well matched output spectrum.

Table 5 gives the input parameters of the best matched model, and the corresponding output spectral fluxes are compared to the observed ones in Table 6.

\section{Discussion}

The best-fit model input parameters are our final values for the characteristics of the PN and its central star.

\subsection{Nebular density and temperature}

The mean nebular electron density from the model turns out to be $\sim 9300 \mathrm{~cm}^{-3}$ which is in good agreement with the value derived from $\mathrm{S}$ II in Table 2. The density from $\mathrm{O}$ II, $\mathrm{Cl}$ III and ArIV are at variance probably due to density variations in the nebula. Alternatively, the measured line fluxes could be in error (i.e., low precision). The observed HST image shows that the surface brightness has a matted finish indicating some degree of clumping. High $\mathrm{S} / \mathrm{N}$ and high resolution spectral observations are needed to establish a more accurate diagnostics. The run of electron density and temperature across the nebula is shown in Fig. 2. The run of electron temperature shows a 
Table 6. The emission line fluxes $(\mathrm{H} \beta=100)$.

\begin{tabular}{|c|c|c|c|c|c|c|c|}
\hline Label & line & Model flux & $\begin{array}{r}\text { Obsd. flux } \\
\text { (dereddened) }\end{array}$ & Label & line & Model flux & $\begin{array}{r}\text { Obsd. flux } \\
\text { (dereddened) }\end{array}$ \\
\hline H 1 & $4861 \mathrm{~A}$ & 100.0 & 100.0 & He 1 & $5876 \mathrm{~A}$ & 12.5 & 9.60 \\
\hline TOTL(O 4) & $1402 \mathrm{~A}$ & 10.3 & 33.5 & O 1 & $6300 \mathrm{~A}$ & 4.20 & 10.7 \\
\hline TOTL(N 4) & $1486 \mathrm{~A}$ & 40.4 & 36.8 & O 1 & $6363 \mathrm{~A}$ & 1.34 & 4.25 \\
\hline TOTL(C 4) & $1549 \mathrm{~A}$ & 714.1 & 502.0 & S 3 & $6312 \mathrm{~A}$ & 2.35 & 2.90 \\
\hline He 2 & $1640 \mathrm{~A}$ & 250.3 & 240.0 & Ar 5 & $6435 \mathrm{~A}$ & 0.713 & 1.11 \\
\hline TOTL(O 3) & $1665 \mathrm{~A}$ & 15.4 & 34.0 & $\mathrm{~N} 2$ & $6584 \mathrm{~A}$ & 276.4 & 225.0 \\
\hline TOTL(N 3) & $1750 \mathrm{~A}$ & 27.3 & 41.0 & S II & $6716 \mathrm{~A}$ & 7.40 & 6.10 \\
\hline TOTL(Si 3) & $1888 \mathrm{~A}$ & 18.0 & 19.8 & S II & $6731 \mathrm{~A}$ & 14.5 & 12.2 \\
\hline TOTL(C 3) & $1909 \mathrm{~A}$ & 625.3 & 628.0 & Ar 5 & $7005 \mathrm{~A}$ & 1.53 & 1.85 \\
\hline TOTL(C 2) & $2326 \mathrm{~A}$ & 99.2 & 129.0 & Ar 3 & $7135 \mathrm{~A}$ & 15.4 & 25.5 \\
\hline $\mathrm{Ne} 4$ & $2424 \mathrm{~A}$ & 43.4 & 116.0 & $\operatorname{Ar} 4$ & $7171 \mathrm{~A}$ & 0.078 & 0.26 \\
\hline $\mathrm{He} 2$ & $2733 \mathrm{~A}$ & 7.85 & 13.0 & $\operatorname{Ar} 4$ & $7237 \mathrm{~A}$ & 0.059 & 0.40 \\
\hline $\mathrm{He} 2$ & $3203 \mathrm{~A}$ & 15.16 & 14.8 & $\operatorname{Ar} 4$ & $7263 \mathrm{~A}$ & 0.065 & 0.20 \\
\hline $\mathrm{Ne} 5$ & $3426 \mathrm{~A}$ & 55.9 & 51.3 & O II & $7323 \mathrm{~A}$ & 10.3 & 11.6 \\
\hline O II & $3726 \mathrm{~A}$ & 96.4 & 100.0 & O II & $7332 \mathrm{~A}$ & 8.63 & 10.9 \\
\hline O II & $3729 \mathrm{~A}$ & 32.2 & 50.0 & Ar 3 & $7751 \mathrm{~A}$ & 3.72 & 4.23 \\
\hline $\mathrm{Ne} 3$ & $3869 \mathrm{~A}$ & 162.2 & 143.0 & $\mathrm{Cl} 4$ & $8047 \mathrm{~A}$ & 1.01 & 0.82 \\
\hline $\mathrm{Ne} 3$ & 3968 A & 48.9 & 51.0 & S 3 & 9069 A & 33.9 & 23.8 \\
\hline S II & $4070 \mathrm{~A}$ & 7.00 & 4.86 & S 3 & $9532 \mathrm{~A}$ & 84.0 & 51.7 \\
\hline S II & $4078 \mathrm{~A}$ & 2.27 & 1.73 & He 2 & $1.012 \mathrm{~m}$ & 9.57 & 3.25 \\
\hline $\mathrm{C} 2$ & $4267 \mathrm{~A}$ & 1.27 & 0.43 & $\operatorname{Mg} 4$ & $4.485 \mathrm{~m}$ & 10.7 & 12.0 \\
\hline H 1 & $4340 \mathrm{~A}$ & 47.01 & 48.2 & Ar 6 & $4.530 \mathrm{~m}$ & 8.91 & 7.95 \\
\hline TOTL(O 3) & $4363 \mathrm{~A}$ & 12.2 & 18.4 & $\operatorname{Mg} 5$ & $5.610 \mathrm{~m}$ & 7.65 & 19.2 \\
\hline $\mathrm{He} 1$ & $4471 \mathrm{~A}$ & 4.08 & 3.30 & Ne 6 & $7.650 \mathrm{~m}$ & 4.44 & 4.10 \\
\hline $\mathrm{Fe} 3$ & $4658 \mathrm{~A}$ & 0.104 & 0.130 & Ar 3 & $9.000 \mathrm{~m}$ & 12.2 & 5.98 \\
\hline He 2 & $4686 \mathrm{~A}$ & 36.3 & 44.5 & S 4 & 10.51 & 90.9 & 100.0 \\
\hline $\operatorname{Ar} 4$ & $4711 \mathrm{~A}$ & 2.18 & 5.80 & $\mathrm{Ne} 2$ & $12.81 \mathrm{~m}$ & 2.69 & 8.08 \\
\hline $\mathrm{Ne} 4$ & $4720 \mathrm{~A}$ & 0.361 & 2.23 & $\mathrm{Ne} 5$ & $14.32 \mathrm{~m}$ & 158.7 & 104.0 \\
\hline $\operatorname{Ar} 4$ & $4740 \mathrm{~A}$ & 3.34 & 7.60 & $\mathrm{Ne} 3$ & $15.55 \mathrm{~m}$ & 189.0 & 166.0 \\
\hline O 3 & $5007 \mathrm{~A}$ & 1581.5 & 1500.0 & S 3 & $18.67 \mathrm{~m}$ & 30.8 & 35.7 \\
\hline Ar 3 & $5192 \mathrm{~A}$ & 0.12 & 0.28 & $\mathrm{Ne} 5$ & $24.31 \mathrm{~m}$ & 66.0 & 90.6 \\
\hline N 1 & $5200 \mathrm{~A}$ & 0.658 & 1.08 & $\mathrm{O} 4$ & $25.88 \mathrm{~m}$ & 211.2 & 266.0 \\
\hline $\mathrm{Cl} 4$ & $5324 \mathrm{~A}$ & 0.017 & 0.130 & $\mathrm{Ne} 3$ & $36.01 \mathrm{~m}$ & 14.4 & 14.4 \\
\hline $\mathrm{Cl} 3$ & $5518 \mathrm{~A}$ & 0.557 & 0.70 & O 3 & $51.80 \mathrm{~m}$ & 75.4 & 74.8 \\
\hline $\mathrm{Cl} 3$ & $5538 \mathrm{~A}$ & 0.887 & 1.40 & N 3 & $57.21 \mathrm{~m}$ & 25.2 & 27.4 \\
\hline $\mathrm{N} 2$ & $5755 \mathrm{~A}$ & 5.35 & 4.50 & O 3 & $88.33 \mathrm{~m}$ & 10.9 & 14.1 \\
\hline
\end{tabular}

Absolute $\mathrm{H} \beta$ flux Model: $2.36 \times 10^{-11} \mathrm{erg} \mathrm{cm}^{-2} \mathrm{~s}^{-1}$; Obsn: $2.34 \times 10^{-11} \mathrm{erg} \mathrm{cm}^{-2} \mathrm{~s}^{-1}$.

Notes: "A" in Col. "Line" signifies Angstrom; "m" signifies $\mu \mathrm{m}$. In Col. "Label", we have followed the notation used by Cloudy for atoms and ions; this will make identifying a line in Cloudy's huge line list easy. Neutral state is indicated by "1" and singly ionized state by "2" etc., "TOTL" typically means the sum of all the lines in the doublet/multiplet; or it could mean sum of all processes: recombination, collisional excitation, and charge transfer. Some elements are represented by usual notation as per Cloudy.

reasonably good agreement with the values obtained from line ratios (Table 3 ).

\subsection{Central star}

The central star is quite hot in our model with $T_{\text {eff }}=159000 \mathrm{~K}$. This compares very well with the Zanstra and Energy Balance temperatures discussed above, but is independent of these determinations. We have used a CSPN model atmosphere with solar composition; If we assume the core mass is $0.6 M_{\odot}$, then for the model $\log g$ of 7.0 , we get $R_{\star}=0.04 R_{\odot}$. This compares well with our earlier estimate from magnitude measurement. We have plotted as Fig. 3 the incident and transmitted continua of the CSPN model atmosphere which we have used. There are several reasons for this. Firstly, the agreement between the predicted and observed line intensities gives not only an estimate of the nebular abundances, but it reflects the accuracy of the CSPN model as well. The incident continuum, although it cannot be observed, can be very useful for future work on the nebula. The transmitted continuum can be observed in the 


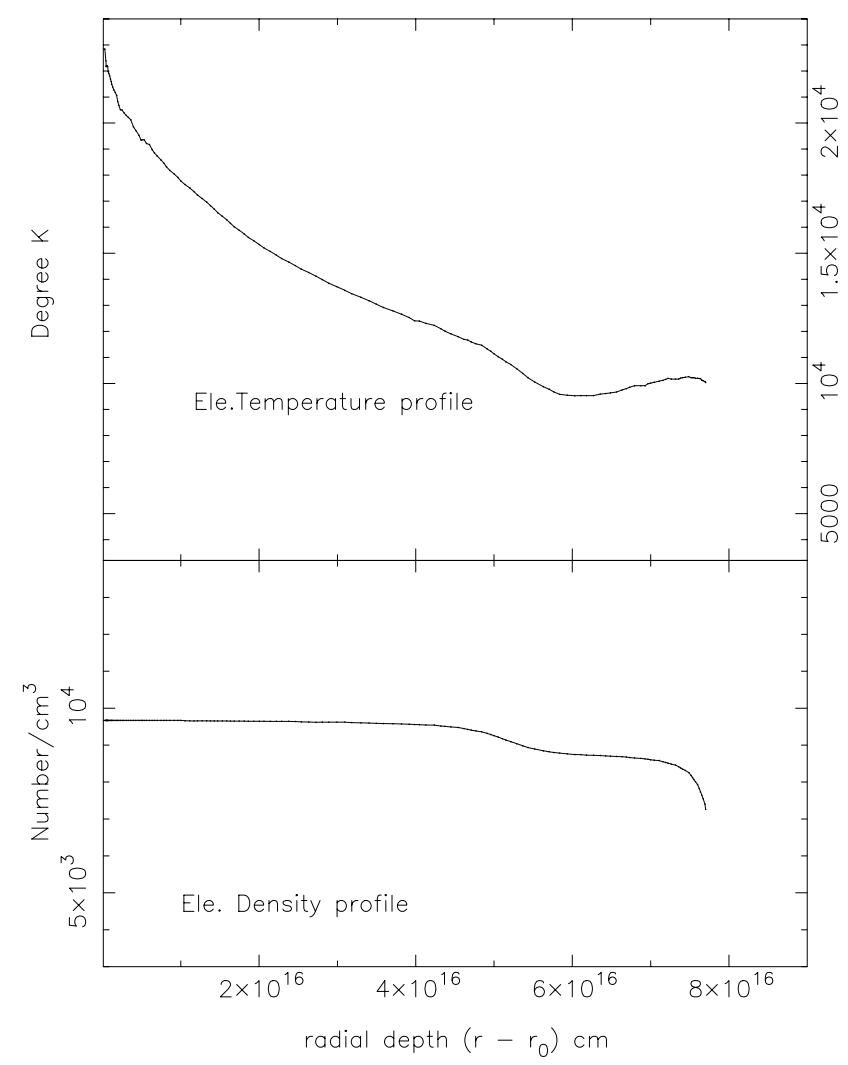

Fig. 2. $T_{\mathrm{e}}$ and $N_{\mathrm{e}}$ across the nebula.

foreseeable future and will be very useful in checking the model. Secondly, from the modeling which has been done it can be seen that the model is energy conserving to a great extent. Only a small amount of radiation is being transmitted through the nebula. This appears to be consistent with the observation mentioned in the introduction that the $21 \mathrm{~cm}$ neutral hydrogen line from the nebula has been measured (Taylor et al. 1990).

\subsection{Comparison of model spectrum with observation}

As mentioned earlier we have attempted to obtain a good match for about 70 observed lines and these are shown in Table 6 . Cloudy computes by default the continuum fluxes at various wavelengths and a very large of number emission lines (nearly 2000 ) in its output spectrum. The notation for line identification is by a label as in Cloudy. This makes identifying any line in Cloudy's huge line list $\left(\sim 10^{6}\right)$ easier (see notes at the bottom of Table 6). The match between model and observation is in general reasonably good. The absolute $\mathrm{H}_{\beta}$ flux from the model is close to what is observed. There are some deviant lines. Neon lines in general behaved rather erratically in all the models we tested and this may be due to uncertainty in the atomic parameters. Some argon lines are also deviant. There are a few observed weak lines of potassium ( $4163 \AA$ and $6102 \AA$ ), found in Hyung et al. (1995), but these lines are not included in Cloudy's default list. Our assumption of a constant density might have been a cause for the deviant behaviour of some lines. Also HST images (Fig. 1) could be interpreted such that the geometry is

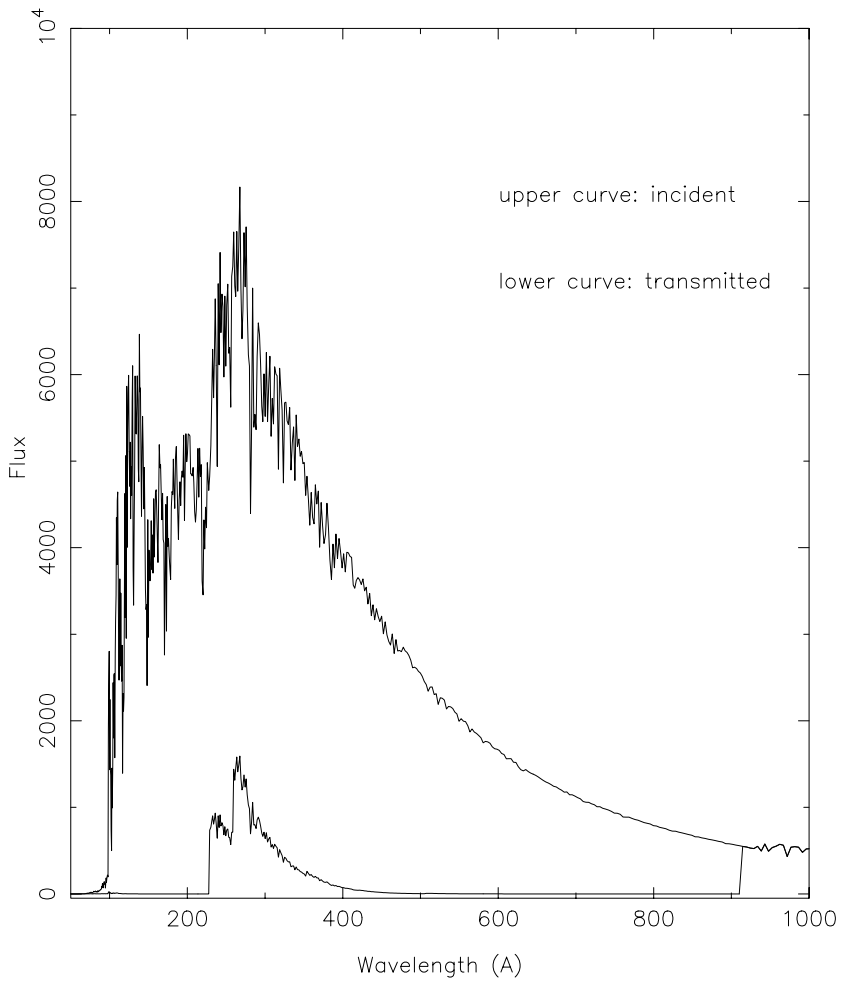

Fig. 3. Stellar ionizing radiation - Incident (line) and transmitted (points); the $y$ axis values are suitably scaled.

rather like a clam with nebular material escaping through an equatorial cleavage, than a simple spherical shell as assumed here.

\section{Nebular abundances}

Barring potassium and calcium, all elements have been represented by at least one line or more in the modeling process. The abundances derived by the two methods are compared in Table 7. The abundances found in the model and those found in the simpler approach are the same within the errors present in the two analyses, in the case of helium, nitrogen, neon, sulphur, chlorine and argon. For the other elements like $\mathrm{Mg}$ and $\mathrm{Si}$, the ionization correction factor is too uncertain for the simpler approach to give accurate results, and we recommend using the abundances found from the model. For K, Ca and Fe only the model abundances are available.

The sulfur abundance is only half that in the sun. Since this element is not produced by any nucleosynthesis reaction in the course of stellar evolution, this must be the primordal abundance of sulfur. In earlier discussions of abundances with ISO data (see papers cited and references therein) sulfur was found to be consistently lower than the solar abundance. Henry et al. (2004) have also pointed out that sulfur is lower than solar for the sample of $85 \mathrm{PN}$ they have studied. They indicate that sulfur also has a lower abundance in PN than in HII regions. However, recent results for galactic HII regions using ISO observations (Martin-Hernandez et al. 2003) show a sulfur abundance which agrees with that of the planetary nebulae. It appears that the solar abundance may be anomalous. 
Table 7. Abundances in NGC 6886.

\begin{tabular}{ccccccc}
\hline \hline Element & $\begin{array}{c}\text { Present } \\
\text { ICF }\end{array}$ & $\begin{array}{c}\text { Present } \\
\text { model }\end{array}$ & $\begin{array}{c}\text { Hyung } \\
\text { ICF }\end{array}$ & $\begin{array}{c}\text { Hyung } \\
\text { model }\end{array}$ & $\begin{array}{c}\text { KH } \\
\text { ICF }\end{array}$ & Solar \\
\hline $\mathrm{He}$ & 0.107 & 0.108 & 0.092 & 0.093 & 0.121 & 0.086 \\
$\mathrm{C}(-4)$ & 14.3 & 19.3 & 4.1 & 4.3 & & 2.5 \\
$\mathrm{~N}(-4)$ & 4.2 & 4.55 & 1.3 & 1.3 & 2.82 & 0.83 \\
$\mathrm{O}(-4)$ & 6.5 & 7.8 & 3.1 & 3.05 & 5.24 & 4.9 \\
$\mathrm{Ne}(-4)$ & 2.0 & 1.7 & 1.0 & 1.62 & 1.22 & 1.2 \\
$\mathrm{Mg}(-5)$ & 2.0 & 3.4 & 0.29 & 0.23 & & 3.8 \\
$\mathrm{Si}(-5)$ & & 2.1 & 2.26 & 1.15 & & 3.5 \\
$\mathrm{~S}(-5)$ & 1.0 & 1.0 & 0.47 & 0.53 & 0.57 & 2.1 \\
$\mathrm{Cl}(-7)$ & 2.2 & 2.2 & 1.82 & 1.7 & 2.2 & 3.2 \\
$\mathrm{Ar}(-6)$ & 2.1 & 2.2 & 1.82 & 1.7 & 4.1 & 2.5 \\
$\mathrm{~K}(-7)$ & & 1.2 & 0.76 & 0.75 & & 1.3 \\
$\mathrm{Ca}(-8)$ & & 1.2 & 20.1 & 20.0 & & 230. \\
$\mathrm{Fe}(-7)$ & & 5.0 & & & & 316. \\
\hline
\end{tabular}

The abundances listed "Hyung" are from Hyung et al. (1995), those listed "KH" from Kwitter \& Henry (2001).

Solar abundances are from Grevesse \& Sauval (1998) except for oxygen and carbon which are from Allende Prieto et al. $(2001,2002)$.

Two earlier abundance determinations are also listed in Table 7. Those of Kwitter \& Henry (2001) are based only on the visual spectrum so that no carbon abundance can be determined. Only one nitrogen line was observed (NII) so that a large ionization correction factor was required, making their nitrogen abundance value very uncertain. Hyung et al. (1995) included the IUE ultraviolet spectrum in their analysis; in fact, the intensities which they find are almost the same as we have used. In discussing the comparison of our abundances with those of Hyung et al. we shall compare separately the determinations using the ICF method and using a model. From the ICF method our abundances are a factor of 3 higher for carbon and nitrogen, a factor of 2 higher for oxygen, neon and sulfur, a factor of 7 higher for magnesium, and essentially the same for argon and chlorine. These differences have nothing to do with the line intensities used since, as already stated, these are very similar. Nor do they have anything to do with the electron density used in the two analyses which are essentially the same. They can be explained (except for magnesium) by different electron temperatures used in the two analyses.

We are capable of obtaining more accurate electron temperatures thanks to the inclusion of the far infrared observations. Eleven temperature determinations are given in Table 4 over a wide range of ionization potentials. Hyung et al. had only two temperature determinations. To make matters worse, they ignore their own two observational determinations and use higher values of temperature "by making use of model predictions".

Abundances have also been determined by Hyung et al. (1995) by modeling the nebula. They claim to be able to reproduce the observations using quite different abundances than we find. From what is said in their article, it appears that their model is rather similar to the one we find. We are at a loss to explain their results. If we use their abundances in our model we cannot reproduce the observational line intensities. We have also tested a model with all the input parameters taken from their work and found the match with observed nebular spectrum to be quite discrepant.

\section{Evolutionary state}

The oxygen abundance is similar to solar and it is therefore likely that the original composition of the star was nearly solar. Both the carbon and nitrogen abundances are now also much greater than solar; these elements were therefore formed in the course of stellar evolution. The large carbon abundance has probably been produced during a third dredge-up, which has also increased helium by a small amount. The third dredge-up does not cause a substantial increase of the nitrogen abundance. The rather high nitrogen abundance was probably produced by hot bottom burning. For this to occur the initial stellar mass has to be about 4 solar masses. This mass gives the best agreement with the abundances found in the evolution models by A. Karakas (thesis: Monash Univ. 2003, supervisor J. Lattanzio).

\section{Conclusion}

We have presented the ISO far infrared spectrum of NGC 6886. Including the ultraviolet and visual spectrum has enabled us to derive nebular abundances of 13 elements. In addition, a simplified nebular model (electron temperature and density) has been found. These have been found both by using the ICF method and by using more elaborate photoionization modeling. The results are given in Tables 4 and 5 and summarized in Table 7. A comparison with earlier work is also given in the last table and shows that our abundances sometimes differ by large factors.

The present temperature and radius of the central star have been obtained and discussed. Its far ultraviolet continuum has also been suggested. Taken together, the nebular abundances and the nebular and stellar parameters suggest that NGC 6886 is probably descended from a star of about 4 solar masses.

Acknowledgements. R.S. would like to acknowledge the use of SIMBAD and ADS for this work.

\section{References}

Acker, A., Ochsenbein, F., Stenholm, B., et al. 1992, Strasb.-ESO Catalogue

Allende Prieto, C., Lambert, D. L., \& Asplund, M. 2001, ApJ, 556, L63

Allende Prieto, C., \& Prieto, D. L. 2002, ApJ, 573, L137

Aller, L. H., \& Czyzak, S. J. 1979, Ap\&SS, 62, 397

Anders, E., \& Grevesse, N. 1989, Geochem. Cosmo., 53, 197

Becker, R. H., White, R. L., \& Edwards, A. L. 1991, ApJS, 75, 1

Benjamin, R. A., Skillman, E. D., \& Smits, D. P. 1999, ApJ, 514, 307

Bernard Salas, J., Pottasch, S. R., Beintema, D. A., \& Wesselius, P. R. 2001, A\&A, 367, 949

Cahn, J. H., Kaler, J. B., \& Stanghellini, L. 1992, A\&AS, 94, 399

Carrasco, L., Serrano, A., \& Costero, R. 1983, RMxAA, 8, 187

Condon, J. J., \& Kaplan, D. L. 1998, ApJS, 117, 361 
Davey, A. R., Storey, P. J., \& Kisielius, R. 2000, A\&AS, 142, 85 Feibelman, W. A. 1994, PASP, 106, 756

Ferland, G. J. 2001, Hazy, a brief introduction to Cloudy 96.04 Fluks, M. A., Plez, B., de Winter, D., et al. 1994, A\&AS, 105, 311

Gregory, P. C., \& Condon, J. J. 1991, ApJS, 75, 1011

Grevesse, N., \& Sauval, A. J. 1998, Sp. Sci. Rev., 85, 161

Griffith, M. R., Burke, B. F., \& Ekers, R. D. 1994, ApJS, 91, 111

Heap, S. R., Corcoran, M., Hintzen, P., et al. 1990, From Miras to PN, ed. Mennessier, \& Omont, 397 (Ed. Frontières)

Henry, R. B. C., Kwitter, K. B., \& Balick, B. 2004, AJ, 127, 2284

Hummer, D. G., \& Storey, P. J. 1987, MNRAS, 224, 801

Hyung, S., Keyes, C. D., \& Aller, L. H. 1995, MNRAS, 272, 49

Kingsburgh, R. L., \& Barlow, M. J. 1994, MNRAS, 271, 257

Kwitter, K. B., \& Henry, R. B. C. 2001, ApJ, 562, 804

Liu, X.-W., Barlow, M. J., Cohen, M., et al. 2001, MNRAS, 323, 343

Leech, K., Kester, D., Shipman, R., et al. 2003, The ISO Handbook Volume V. SWS - The Short Wavelength Spectrometer, ESA SP-1262

Martin-Hernandez, N. L., v.d. Hulst, J. M., \& Tielens, A. G. G. M. 2003, A\&A, 407, 957

Milne, D. K., \& Aller, L. H. 1982, A\&AS, 50, 209

Pottasch, S. R., \& Acker, A. 1989, A\&A, 221, 123

Pottasch, S. R., Wesselius, P. R., Wu, C. C., et al. 1977, A\&A, 54, 435
Pottasch, S. R., Dennefeld, M., \& Mo, J.-E. 1986, A\&A, 155, 397

Pottasch, S. R., \& Beintema, D. A. 1999, A\&A, 347, 974

Pottasch, S. R., Beintema, D. A., \& Feibelman, W. A. 2000, A\&A, 363, 767

Pottasch, S. R., Beintema, D. A., Bernard Salas, J., \& Feibelman, W. A. 2001, A\&A, 380, 684

Pottasch, S. R., Preite-Martinez, A., Olnon, F. M., et al. 1986, A\&A, 161, 363

Pottasch, S. R., Beintema, D. A., Bernard Salas, J., et al. 2002, A\&A, 393, 285

Preite-Martinez, A., \& Pottasch, S. R. 1983, A\&A, 126, 31

Rauch, T. 2003, A\&A, 403, 709

Roche, P. F., \& Aitken, D. K. 1986, MNRAS, 221, 63

Rowlands, N., Houck, J. R., Skrutskie, M. F., et al. 1993, PASP, 105, 1287

Seaton, M. J. 1979, MNRAS, 187, 73

Surendiranath, R., Pottasch, S. R., \& García-Lario, P. 2004, A\&A, 421, 1051

Taylor, A. R., Gussie, G. T., \& Pottasch, S. R. 1990, ApJ, 351, 515

Tsamis, Y. G., Barlow, M. J., Liu, X.-W., et al. 2003, MNRAS, 345, 186

Wang, W., Liu, X.-W., Zhang, Y., \& Barlow, M. J. 2004, A\&A, 427, 873 